\title{
MOBILE COMPLEX FOR RAPID DIAGNOSIS OF THE TECHNOLOGICAL SYSTEM ELEMENTS
}

\author{
Alexey Gavrilin*, Boris Moyzes, Alexzander Cherkasov, Kirill Mel'nov, Xiaoliang Zhang \\ Tomsk Polytechnic University, Institute of Cybernetics, 634050, Tomsk, Russia
}

\begin{abstract}
The article shows the up-to-dateness of the new informing and measuring tools and technologies development. It is reviewed the mobile complex for runtime diagnostics of technological system "machine-toolinstrument-detail". It was found that the use of the complex allows to identify the frequency area in which the appearance of resonance of the technological system elements is possible, and thus to draw a conclusion on the technical state of the diagnosed object. It is concluded that there is the prospects for the use of the above mentioned mobile complex for vibration diagnostics.
\end{abstract}

\section{Introduction}

The vibration that occurs in the production equipment, leads not only to a positive effect, useful for various technological tasks [1-8], but also negative effect. In terms of metal working vibration can have a significant effect on the accuracy of the equipment operation of (accuracy and surface roughness), the reliability of its operation, the protection level of installed in close proximity the precision manufacturing equipment, the safety of the personnel on the consequences of possible emergency situations.

The development of scientific and technological progress and the requirements for the processingaccuracy has set the development trends of metal working equipment design, the main ones are:

- increased productivity through increased automation and intensification of the cutting modes (high-speed cutting);

- improved accuracy of processing [9], including the vibration damping [10] and the choice of optimal operating regime [10];

- improved reliability equipment operation due to on-time diagnostics [9], including vibration diagnostics to determine vibration activity of the technological system elements which negatively affect the working accuracy of workpiece and tool life.

The development of the metal-working equipment designs led to the further development of information and measurement technology [11-14] for vibration diagnostics of the technological system "machine-tool-tool-instrument-piece".

Diagnostic facilities can be both mobile and stationary, but the stationary ones cannot be used for fast diagnostics of metalworking equipment. It should be noted that there is a wide range of parts in appliances.

\footnotetext{
* Corresponding author: tom-gawral@list.ru
} 
There are known the designs of the mobile diagnostic complexes of foreign and domestic origin as well as their application techniques [11-14]. These complexes show good results, but they are complicated to operate (instruction and special skills are required) that determined the necessity to develop an up-to-date, compact, reliable and easy-to-use diagnostic system for vibration diagnostics.

\section{Mobile complex for rapid diagnosis of the technological system elements}

The scientists of Tomsk Polytechnic University in collaboration with the company "VITEK Siberia" (Tomsk) has developed and tested the diagnostic system [10], consisting of three modules (Figure 1):

- piezoelectric sensors;

- vibration and measurement unit;

- a laptop with the operating software.

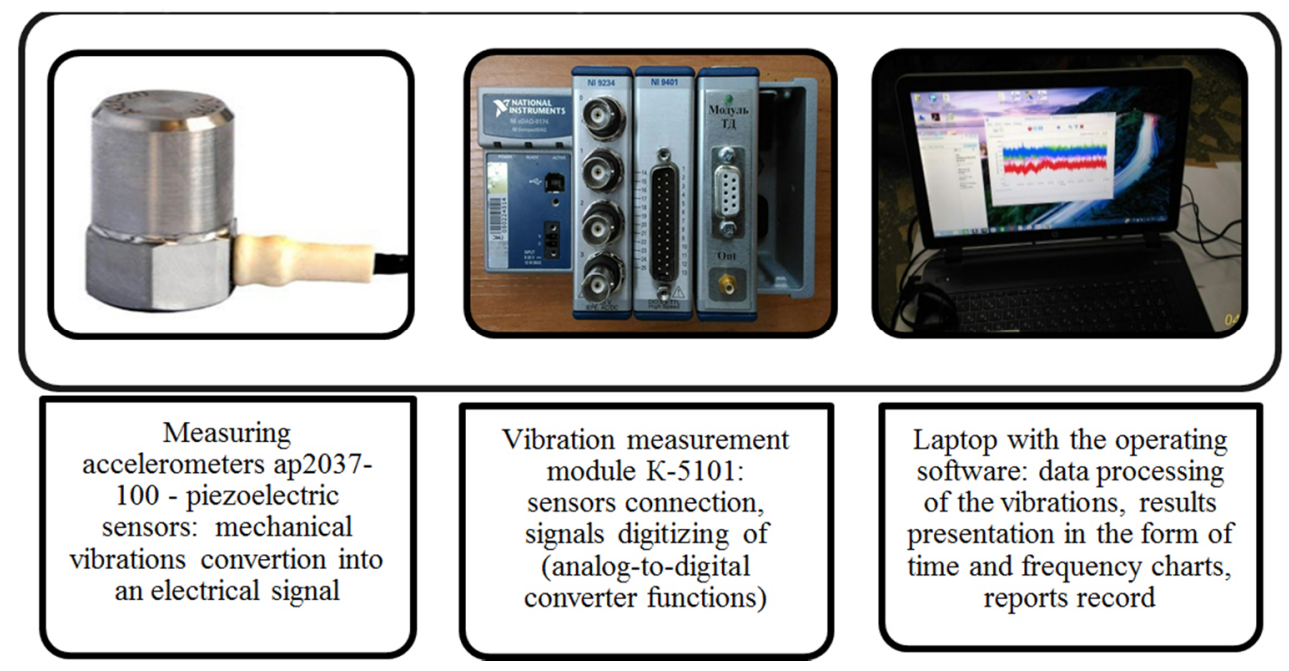

Figure 1. Modules of the diagnostic complex.

It was required for the diagnostic complex to be competitive on the market which determined modification of the software in the follow terms:

- presentation of the diagnostic results - the applicability of the first-fifth orders filters of the low and high frequency as well as band and-reject, which allows to adapt to different conditions faster (Figure 2) are added;

- signal processing - frequency band of the amplitude spectrum selection for the vibration pattern analysis (Figure 3) was simplified;

- channel information selection - up to four or more. 


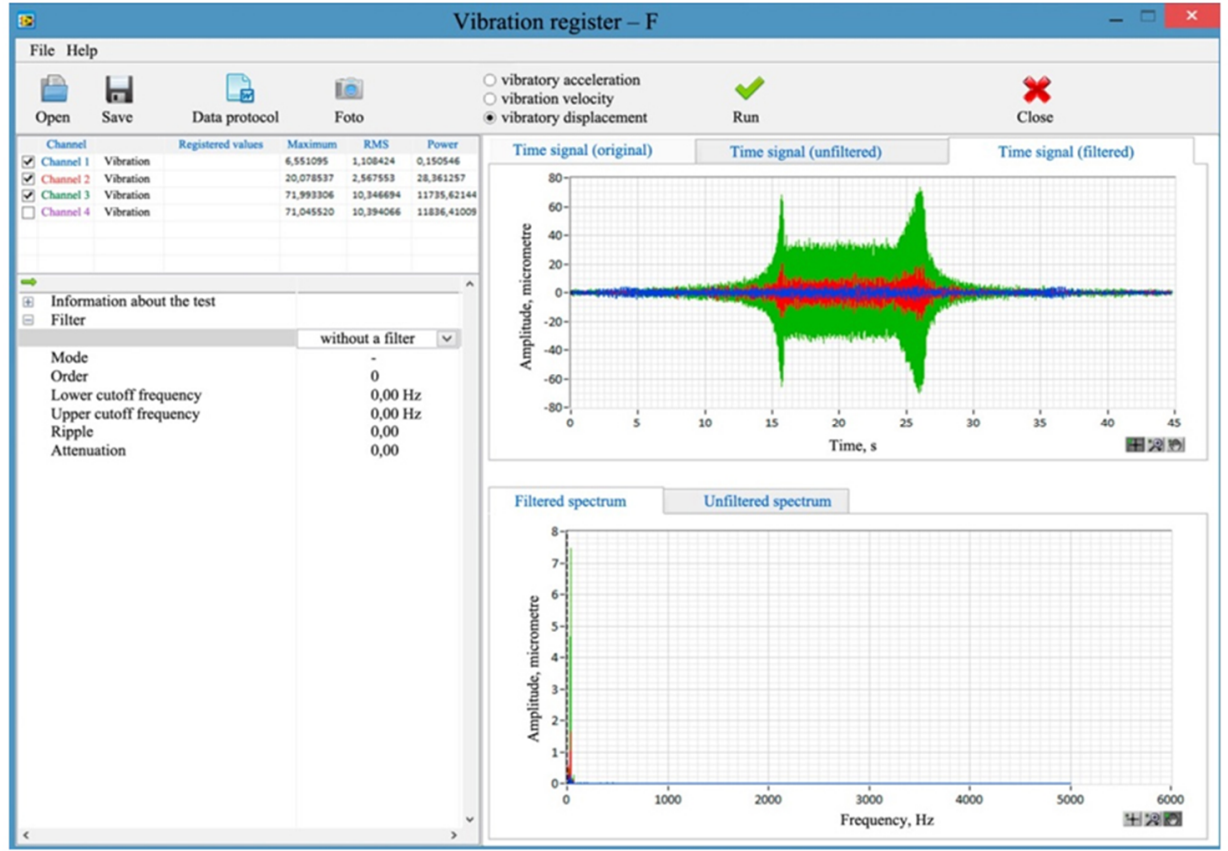

Figure 2. Software window fragment of "Vibration register - F": left - channel information selection and filtering parameters settings; right - signal waveform by three channels when accelerating to $2000 \mathrm{rev} / \mathrm{min}$, dwelling and braking and its spectrum.

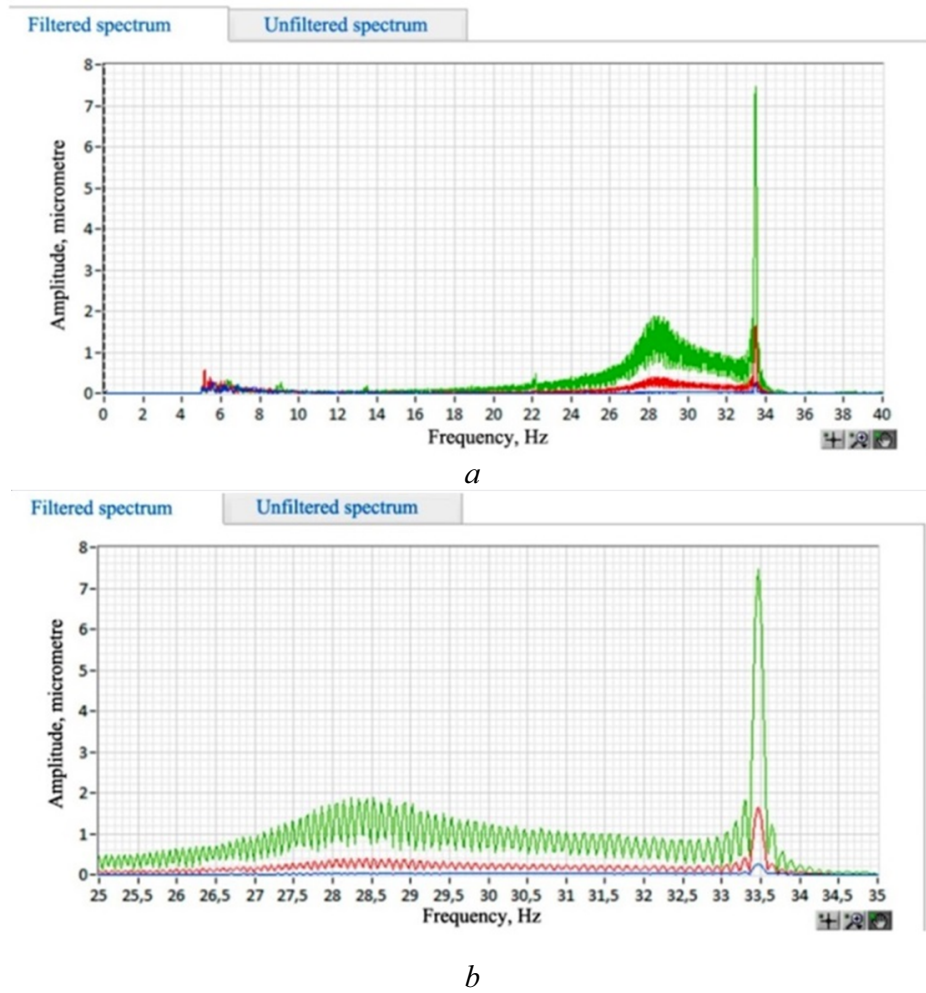

Figure 3. Software window fragments of "Vibration register $-F$ " for different scales: $a-1 \mathrm{~Hz} ; b-0.5 \mathrm{~Hz}$. 


\section{The diagnostics procedure by the mobile diagnostic complex}

Testing of the upgraded diagnostic complex and the procedure development was assigned to students as part of the educational process to simplify the use of the diagnostic complex while testing.

\subsection{The diagnostic complex setting (basic diagnostic scheme development)}

The complex setting includes the follow steps:

- the selection of the diagnosing unit and fixing the vibration directions;

- the selection of bonding technique and a sensing point;

- the connection complex modules to each other

The screwing lathe 16K20 model was chosen for researching. Sensors layout is shown in Figure 4 (magnet fastening).

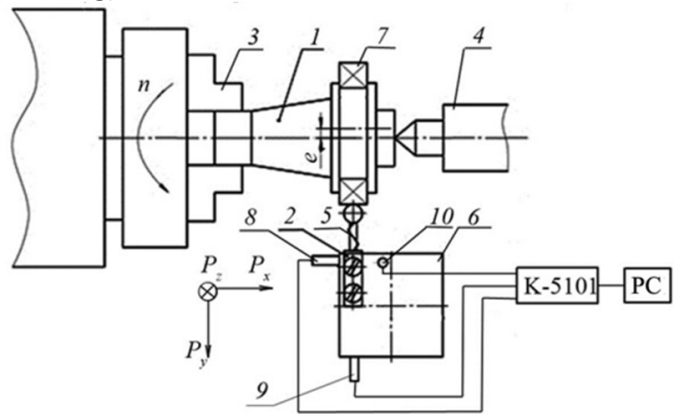

Figure 4. The basic diagram of the complex setting: 1 - wobbler shaft; 2 - holder; 3 - three-jaw chuck; 4 -tail block; 5 -elastic element; 6 -toolholder; 7 - bearing part; 8 -10-accelerometers; K-5101 - vibration measurement module; $\mathrm{PC}-$ a laptop; $P_{x}, P_{y}, P_{z}$ - operative directions of cutting force components.

Sensors are placed on the toolholder (due to the inability to install them directly on the spindle of the machine-tool), the axes $X, Y$ and $Z$ are the directions of vibration registration, $P_{x}, P_{y}, P_{z}$ - operative directions of exciting cutting force components.

Excitation was performed during rotating spindle due to the eccentricity $e$ by the elastic element 5 (by spring- actuated piston plunger), mounted in the holder 2 , and fastened to the toolholder 6 . The elastic element 5 , acting through the bearing 7 on the shaft 1 , loads the spindle by the harmonically varying force.

\subsection{Complex setting}

To set the complex the following steps are required:

- to identify the sensor channels on the computer (Figures 2, 4):

○ channel 1 (blue) - the axis $X$;

- channel 2 (red) - the axis $Z$;

- channel 3 (green) - the axis $Y$;

- to configure the software - select:

○ registered values: vibration displacement (selected), vibration velocity, vibration acceleration;

○ tool: cutter (selected), the miller;

$\circ$ the filter type and its parameters etc.

- to configure the software - select: 
○ registered values: vibration displacement (selected), vibration velocity, vibration acceleration;

○ tool: cutter (selected), the miller;

$\circ$ the filter type and its parameters etc.

\subsection{The modes selection and the test operation plan development}

As a power impact source the spring-loaded plunger rod has been chosen (Figure 4). Spindle rotation speed is $2000 \mathrm{rev} / \mathrm{min}$ (maximum).

Experimental plan: to register the vibration patterns during acceleration, dwelling and breaking.

\subsection{Starting the diagnosing object and registering the results}

Vibrations pattern obtained during the experiment is shown in Figure 2.

\subsection{Report making}

The test results are generated automatically in Microsoft Word format with the time and spectral diagrams.

\subsection{The data analysis and recommended guideline}

Special software allows to choose a specific time range (Figure 5, a) for reviewing and analyzing and the corresponding signal spectrum (Figure $5, b$ ), namely a sharply increased amplitude fluctuations zone.

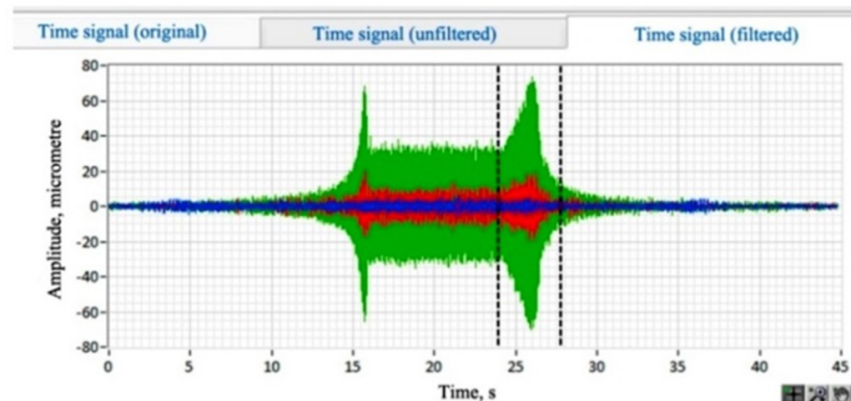

$a$

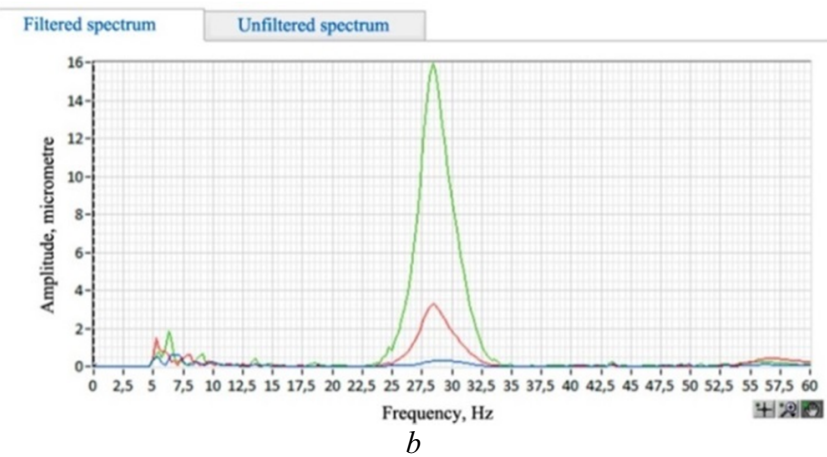

Figure 5. Software window fragment of "Vibration register $-F$ ":

$a$-waveform through the three channels with the time span selection between 24-28 $\mathrm{s} ; b$-signal spectrum. 
Vibration pattern (Figure 5) shows the presence of oscillation amplitude jump at a frequency of $33.45 \mathrm{~Hz}$, which is close to the disturbing frequency at the spindle:

$$
f_{\mathrm{df}}=\frac{n_{\mathrm{sp}}}{60}=\frac{2000}{60}=33.3 \mathrm{~Hz},
$$

where $n_{\mathrm{sp}}-$ spindle speed, rev/min.

\section{Conclusion}

In addition it is possible to determine the frequencies close to resonance, according to the obtained values of the vibratory displacement (Figure 2) as well as the crest factor value can be determined, as the ratio of maximum amplitude value of time signal to RMS (Table 1).

Table 1. Measurement results of vibratory displacement, RMS and crest factor calculation.

\begin{tabular}{|c|c|c|c|}
\hline Channel & Maximum & RMS & Crest factor \\
\hline 1 & 6,55 & 1,11 & 5,9 \\
\hline 2 & 20,08 & 2,57 & 7,81 \\
\hline 3 & 71,99 & 10,35 & 6,96 \\
\hline 4 & 71,05 & 10,39 & 6,84 \\
\hline
\end{tabular}

Increased values of cross-factor (above 1.41) indicate the presence of impact in the drive elements, which requires thorough diagnostic.

Thus, the mobile complex for fast diagnosis of the technological system elements with improved special software allows to optimize the signal processing in the terms of filtration and frequency analysis, and the research carried out by students, has demonstrated how simple to operate the complex.

\section{References}

[1] A.I. Nizhegorodov, A.N. Gavrilin, B.B. Moyzes, Key Eng. Mater. 685, 320 (2015) doi: 10.4028/www.scientific.net/KEM.685.320

[2] A.I. Nizhegorodov, A.N. Gavrilin, B.B. Moyzes, J. Phys: Conf. Ser. 671, 012037 (2016) doi: 10.1088/1742-6596/671/1/012037

[3] R.M. Mustafina, I.A. Plotnikov, I.V. Plotnikova, O.N. Tchaikovskaya, J. Phys: Conf. Ser. 671, 012046 (2016) doi: 10.1088/1742-6596/671/1/012046

[4] A.Y. Petrova, O.N. Chaikovskaya, I.V. Plotnikova, Tech. Phys. J. 60, 592 (2015) doi: $10.1134 / \mathrm{S} 1063784215040222$

[5] A.P. Surzhikov, E.N. Lysenko, V.A. Vlasov, A.V. Malyshev, E.V. Nikolaev, RUSS PHYS J. 56, 681 (2013) doi: 10.1007/s11182-013-0085-6

[6] O.V. Vasileva, Y.N. Isaev, A.A. Budko, A.I. Filkov, J. Phys.: Conf. Ser. 552, 012026 (2014) doi: 10.1088/1742-6596/552/1/012026

[7] I. Plotnikova, L. Redko, O. Titova, O. Chaykovskaya, IOP Conf. Ser.: Mater. Sci. Eng. 132, 012023 (2016) doi : 10.1088/1757-899X/132/1/012023

[8] S. Obukhov, I. Plotnikov, M. Kryuchkova, IOP Conf. Ser.: Mater. Sci. Eng. 132, 012017 (2016) doi : 10.1088/1757-899X/132/1/012017

[9] A.M. Dalsky, Reference-book of technologist-mechanical engineer (Mashinostroenie, Moscow, 2003)

[10] A. Gavrilin, B. Moyzes, O. Zharkevich, JVE 17, 3495 (2015)

[11] C. Grigoriev, M. Kozochkin, F. Sabirov, V. Sinopalnikov, Vestnik MSTU STANKIN 4, 27 (2010) 
[12] M. Kozochkin, A. Privalov, Instrum. Syst.: Monit., Control and Diagnostics 2, 18 (2013)

[13] M. Kozochkin, F. Sabirov, Vestnik MSTU STANKIN 3, 14 (2008)

[14] D. Hartono, D. Halim, A. Widodo, G.W. Roberts, MATEC Web of Conferences 65, 01003 (2016) doi: 10.1051/matecconf/20166501003 